\title{
Atenção à saúde do portador de tuberculose: atuação do Agente Comunitário de
}

\section{Saúde: revisão integrativa}

Attencion to the health of tuberculosis patients: performance of the Community Health Agent: integrative review

Atención a la salud del paciente com tuberculosis: Agente de Salud Comunitário: revisión

Luiza Cristina de Souza Brandão Correia ORCID: https://orcid.org/0000-0001-7751-5692 Universidade Ceuma, Brasil E-mail: luicristina@hotmail.com

Ahmed Ali Gomes Yassin

ORCID: https://orcid.org/0000-0002-7546-7452 Universidade Ceuma, Brasil

E-mail:ahmedyassin715@gmail.com

Débora Alessandra Hickmann Ayres ORCID: https://orcid.org/0000-0002-8399-9387 Universidade Ceuma, Brasil

E-mail:debora_hicjmann@hotmail.com

Ramon Abreu de Lucena

ORCID: https://orcid.org/0000-0002-8302-8732 Universidade Ceuma, Brasil E-mail:ramon9al@gmail.com

Maria Vitória Rodrigues Passarinho ORCID: https://orcid.org/0000-0003-0502-2332 Universidade Ceuma, Brasil e-mail: mvrodrigues2096@icloud.com

Mônica Andrea Miranda Aragão ORCID: https://orcid.org/0000-0002-7689-9070 Universidade Ceuma, Brasil

E-mail: monica.miranda@ceuma.com.br

\begin{abstract}
Resumo
Objetivo: Destacar a importância da atuação do agente comunitário de saúde na atenção ao portador de tuberculose. Metodologia: Trata-se de uma revisão integrativa de literatura. A busca na literatura foi realizada nas seguintes bases de dados científicos: Scientific Eletronic Libary Online (Scielo), Biblioteca Virtual em Saúde (BVS) e Google Acadêmico. Resultados: Acerca da atenção Primária ao portador de tuberculose foram selecionados 12 artigos sendo publicados entre os anos de 2017 a 2020, constatado que 8 artigos abordavam o tema em questão e esses escolhidos como a fonte de pesquisa. Conclusão: Pode-se inferir que a contribuição do agente comunitário de saúde no acompanhamento e promoção da saúde do portador de tuberculose é indispensável, desde a captação, identificação, acompanhamento do tratamento, mostrando em concordância com as literaturas abordadas. Revelam-se das muitas dificuldades existentes, que há muito a ser feito e que o investimento em conhecimento e ações precisam ser enfatizados, demandando atenção e doação dos profissionais a frente dessa patologia.
\end{abstract}

Palavras-chave: Agente comunitário de saúde; Saúde primária; Tuberculose.

\begin{abstract}
Objective: To highlight the importance of community health agents in the care of tuberculosis patients. Methodology: This is an integrative literature review. The literature research was performed in the following scientific databases: Scientific Electronic Libary Online (Scielo), Virtual Health Library (VHL) and Google Scholar. Results: Regarding the primary care of tuberculosis patients, 12 articles were selected, these been published between 2017 to 2020, it was found that 8 articles addressed the topic in question and these were chosen as the source of research. Conclusion: It can be inferred that the contribution of the community health agent in monitoring and promoting the health of tuberculosis patients is indispensable, from the approach, identification, monitoring of treatment, showing that it is in agreement with the literature discussed. The many existing difficulties reveal that there is a lot to be done and that investment in
\end{abstract}


knowledge and actions need to be emphasized, demanding attention and dedication from professionals facing this pathology.

Keywords: Community health agent; Primary health; Tuberculosis.

\section{Resumen}

Objetivo: Resaltar la importancia del rol de los agentes comunitarios de salud en la atención al paciente tuberculoso. Metodología: Se trata de una revisión integradora de la literatura. La búsqueda bibliográfica se realizó en las siguientes bases de datos científicas: Scientific Electronic Libary Online (Scielo), Virtual Health Library (BVS) y Academic Google. Resultados: Sobre la atención primaria al paciente tuberculoso, se seleccionaron y publicaron 12 artículos entre los años 2017 a 2020, se encontró que 8 artículos abordaron el tema en cuestión y estos fueron elegidos como fuente de investigación. Conclusión: Se puede inferir que el aporte del agente de salud comunitaria en el seguimiento y promoción de la salud de los pacientes tuberculosos es fundamental, a partir de la captación, identificación, seguimiento del tratamiento, demostrando que está de acuerdo con la literatura discutida. Las múltiples dificultades existentes revelan que queda mucho por hacer y que es necesario enfatizar la inversión en conocimientos y acciones, exigiendo la atención y la donación de los profesionales que enfrentan esta patología.

Palabras clave: Agente de salud comunitaria; Salud primaria; Tuberculosis.

\section{Introdução}

Segundo o Ministério de Saúde a tuberculose (TB) continua sendo mundialmente um importante problema de saúde, exigindo o desenvolvimento de estratégias para o seu controle, considerando aspectos humanitários, econômicos e de saúde pública (Brasil, 2019).

A Tuberculose é uma doença infectocontagiosa e transmissível que afeta prioritariamente os pulmões. Anualmente, são notificados cerca de dez milhões de novos casos em todo o mundo, levando mais de um milhão de pessoas a óbito (Fernandes, et al, 2020).

O relatório Mundial de Tuberculose 2018 da Organização Mundial da Saúde, traz que cerca de 10 milhões de casos com uma morte a cada segundo são registrados mundialmente. No Brasil nesse mesmo ano foram 76 mil casos e 45 mil mortes.

No Brasil, em 2019, foram registrados 73.864 mil casos novos da doença. Mesmo com cura, o abandono do tratamento é o principal motivo para a ocorrência de vítimas fatais. Apesar da redução de $8 \%$ no número de óbitos na última década (4.881 óbitos em 2008), em 2018, 4.490 pessoas morreram no país. O Maranhão traz registrados 2.623 no ano de 2019, segundo o Data SUS, com 162 casos na cidade de Imperatriz são registrados nesse mesmo ano (Brasil, 2019; Brasil, 2020).

A Tuberculose é a terceira causa de morte por doença infecciosa na população geral e a primeira causa de morte entre as doenças infecciosas definidas nas pessoas que vivem com HIV/AIDS. Esta realidade levou à inclusão da TB entre as sete prioridades estabelecidas no Programa de Pesquisa e Desenvolvimento em Doenças Negligenciadas (Melo, 2020).

$\mathrm{O}$ tratamento da tuberculose permanece um desafio em função da necessidade de que, em sua abordagem, seja considerado o contexto da saúde do indivíduo e da saúde coletiva. Adicionalmente, as questões sociais e econômicas têm-se mostrado como variáveis a serem consideradas na efetividade do tratamento.

O agente comunitário de saúde é um importante agente social na equipe de saúde da família na busca ativa da detecção de casos suspeitos ou confirmados e acompanhamento de saúde na linha de cuidado da tuberculose. O Agente comunitário de saúde é um importante agente de vigilância no controle das doenças epidemiológicas através da perspectiva da busca ativa de casos como a Tuberculose que é uma doença infecciosa transmissível e embora seja uma doença tratável e curável ainda existem muitos estigmas e preconceito (Rizzo, 2020).

A educação permanente é uma prática transformadora e significativa no campo do saber e das práticas de saúde, ao qual, vem construindo para a qualificação profissional de todos os envolvidos e transformações de realidades no campo da saúde da família propiciando a reflexão coletiva sobre o trabalho e ações a serem desenvolvidas (Rizzo, 2020).

Diante do exposto, o presente estudo tem como propósito levantar reflexões acerca da importância do agente comunitário de saúde no enfrentamento da tuberculose, seus atributos essênciais, sua atuação, as dificuldades no enfrentamento, 
acompanhamento desde do diagnóstico até a alta médica e a capacitação desses profissionais, bem como a utilização dos recursos humanos, materiais técnicos e científicos para o acompanhamento e tratamento da tuberculose.

\section{Metodologia}

Trata-se de uma revisão integrativa da literatura, na busca de pesquisas relevantes sobre o agente comunitário de saúde e sua abordagem ao portador de tuberculose.

Segundo Souza (2010), a revisão integrativa obedece às fases na sua elaboração: iniciando pela identificação do tema e a formulação da questão da pesquisa; após segue-se para o estabelecimento de critérios de inclusão e exclusão dos estudos para amostragem; e por final a coleta dos dados que serão extraídos do material antes selecionados.

Seguindo as fases antes mencionadas seguiu-se primeiramente com a formulação de pergunta norteadora: Qual a importância do Agente Comunitário de Saúde no manejo dos pacientes portadores de tuberculose no âmbito da atenção primária à saúde?

A busca na literatura foi realizada nas seguintes bases de dados: Scientific Electronic Library Online (SCIELO), Biblioteca Virtual em Saúde (BVS) e Google Acadêmico, utilizando como palavras de busca, tuberculose, agente comunitário de saúde e educação em saúde. Como critérios de inclusão foram selecionados artigos publicados em português com produção e publicação do período de 2017 a 2021 e como critérios de exclusão aqueles os quais não apresentavam a relação entre a tuberculose e os agentes comunitários de saúde e que tenham produção e publicação realizadas antes do ano 2017 (Fluxograma 1).

Os artigos foram lidos na íntegra para melhor tomada de decisão na inclusão e exclusão, com um total de 12 artigos que foram avaliados com uma visão crítica de cada pesquisador de maneira individual e independente com suas devidas seleções, os mesmos diante das suas discordâncias entraram em consenso.

$\mathrm{Na}$ análise do material foram coletados dados referentes ao periódico (título, ano de publicação), aos autores (nomes completos) e ao estudo (objetivo, vinculação acadêmica, referencial teórico, tipo de estudo, aspectos metodológicos, resultados e recomendações). Os dados da interpretação passaram por uma avaliação dos artigos selecionados, comparando a teoria, e observando as conclusões e implicações resultantes da revisão integrativa. Após a avaliação crítica, obteve-se uma amostra final de 8 artigos. Para minimizar as discordâncias a avaliação e a seleção dos estudos foi realizada por 4 componentes do estudo, com uma discussão final para consenso de inclusão e exclusão dos materiais utilizados para o estudo e pesquisa. 
Fluxograma 1. Fluxograma do processo de escolha dos artigos.
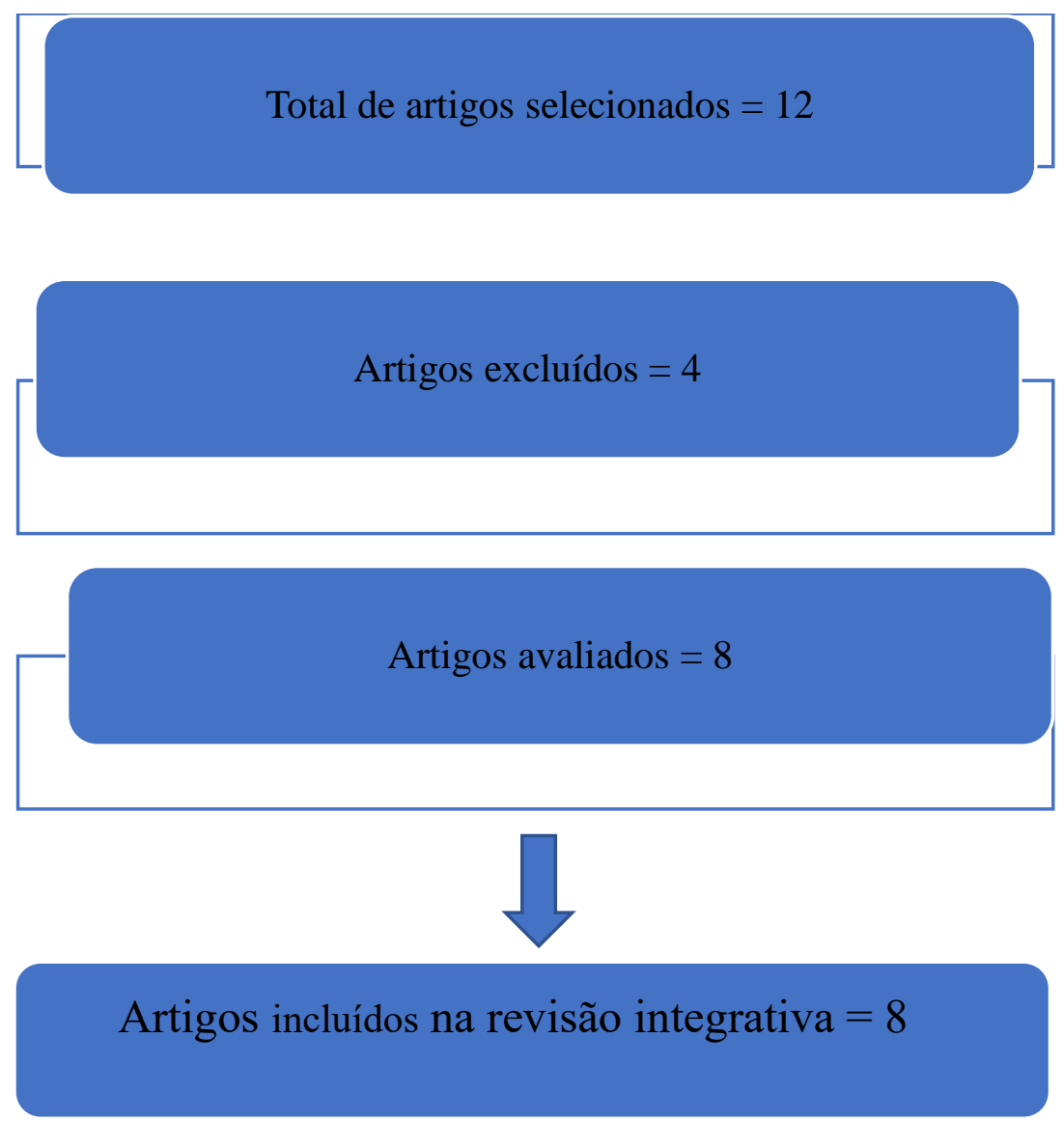

\section{Resultados e Discussão}

A amostra inicial foi composta por 12 artigos encontrados na base de dados pesquisadas com publicação dos anos de 2017 a 2020, dos quais 5 foram excluídos por não contemplarem os critérios de abordagem relacionado aos agentes comunitários e sua atuação na tuberculose. Assim, 8 artigos foram lidos na íntegra e utilizados para realização dessa pesquisa, os quais estão dispostos a seguir (Quadro 1).

Quadro 1. Distribuição das publicações referente a tuberculose e ação dos agentes comunitários de saúde. Imperatriz/Ma, 2021.

\begin{tabular}{|c|c|c|c|}
\hline Número & Título & Autor & Ano \\
\hline 1 & $\begin{array}{l}\text { Agentes comunitários de saúde na atenção primária no Brasil: } \\
\text { multiplicidade de atividades e fragilização da formação }\end{array}$ & SAMUDIO, J. L.P, et al. & 2017 \\
\hline 2 & $\begin{array}{l}\text { Os agentes comunitários na Atenção Primária à Saúde no Brasil: } \\
\text { inventário de conquistas e desafios }\end{array}$ & $\begin{array}{l}\text { MOROSINI, M. V; FONSECA, A } \\
\text { M. }\end{array}$ & 2018 \\
\hline 3 & $\begin{array}{l}\text { Conhecimento, atitudes e práticas de agentes comunitários de saúde } \\
\text { sobre tuberculose pulmonar em uma capital do Nordeste do Brasil }\end{array}$ & GASPAR, L.M.S, et al. & 2018 \\
\hline 4 & $\begin{array}{l}\text { Capacitação dos agentes comunitários de saúde de unidade básica } \\
\text { de saúde como estratégia para detecção de casos de tuberculose. }\end{array}$ & BATISTA, L. D. A. & 2018 \\
\hline 5 & $\begin{array}{l}\text { Capacitação dos Agentes Comunitários com enfoque em } \\
\text { Tuberculose na Atenção Básica }\end{array}$ & MAUÉS. G. M, et al & 2020 \\
\hline 6 & $\begin{array}{l}\text { O tratamento da tuberculose sob a ótica dos profissionais da Atenção } \\
\text { Primária à Saúde }\end{array}$ & SIQUEIRA, T.C et al & 2020 \\
\hline 7 & $\begin{array}{l}\text { Intervenção educativa sobre tuberculose para agentes comunitários } \\
\text { de saúde em unidades de atenção primária à saúde em um município } \\
\text { da região Norte }\end{array}$ & FREITAS P. R, et al & 2020 \\
\hline 8 & $\begin{array}{l}\text { Agentes sociais na Estratégia Saúde da Família (ESF) para o } \\
\text { controle da Tuberculose: Educação permanente para os Agentes } \\
\text { Comunitários de Saúde (ACS): uma revisão integrativa }\end{array}$ & RIZZO,E.R et al & 2021 \\
\hline
\end{tabular}

Fonte: Autores (2021). 


\section{Discussão}

Da leitura e análise das publicações, ascenderam quatro 3 temas relevantes: Tema 1: Importância do agente comunitário de saúde na atenção ao portador de tuberculose; tema 2: Desafios na prevenção e tratamento da tuberculose; 3: Capacitação dos agentes comunitários de saúde no manejo da tuberculose.

\section{Tema 1: Importância do Agente Comunitário de Saúde na atenção ao portador de tuberculose}

Segundo Freitas e colaboradores (2020), a atenção Primária à Saúde, alicerce central do Sistema Único de Saúde, é a principal aliada na luta contra a tuberculose, por meio da Estratégia de Saúde da Família (ESF), tendo em vista que a diminuição da incidência e do abandono do tratamento da TB estão intimamente relacionados com a cobertura da equipe de saúde da família e o desenvolvimento de suas atribuições.

Na atenção à Tuberculose, Freitas (2020), relata que o agente comunitário de saúde tem o papel de identificar os sintomáticos respiratórios nos domicílios e na comunidade e encaminhá-los juntamente com os contatos para avaliação nas unidades de saúde. Devem orientar quanto à importância da realização dos exames diagnósticos e a coleta/encaminhamento do escarro para análise laboratorial, bem como supervisionar a tomada de medicação e realizar a busca de faltosos e de pacientes que abandonaram o tratamento (como citado em, Brasil, 2011a; Brasil, 2011b).

Rizzo e colaboradores (2021), afirma que os agentes comunitários de saúde estão presentes em diversos contextos geográficos e culturais, realizando ações que objetivam o cuidado à saúde da comunidade. Seguem orientações de acordo com cada sistema de saúde e demandas contextuais. No entanto percebe-se a necessidade de clareza quanto às suas atribuições e respectiva execução. Também é preciso que haja mais esclarecimentos sobre as habilidades e competências de mandadas pelo cuidado em saúde, associadas à inventividade, para que a saúde da comunidade seja seu foco.

O autor afirma ainda que a valorização do agente comunitário de saúde é importante para na motivação no processo de trabalho para as mudanças de formação das práticas que atenda tal demanda, é necessário promover mudanças nos processos de formação, consubstanciadas pela atuação qualificada e valorização em um contexto permanente de educação profissional.

O estudo de Samúdio e colaboradores (2017), traz que o agente comunitário de saúde é personagem fundamental pela proximidade dos problemas da comunidade e pela facilidade em se comunicar e exercer liderança, destaca ainda sua atuação como facilitador, dada a possibilidade de adentrar na intimidade das famílias, percebendo suas necessidades e as da comunidade, firmando assim o seu papel como apoio social e mediador de conflitos na comunidade.

Destaca-se a atuação com redes de relacionamentos significativos, como família, amizade, trabalho, estudo de comunidade, além de agências sociais e institucionais. Tal proximidade contribui para a articulação e o fortalecimento de relações entre pessoas de um território e sistemas de cuidado (Samúdio, 2017).

\section{Tema 2: Desafios enfrentados pelos agentes comunitários de saúde no manejo do paciente com tuberculose}

O desconhecimento do agente comunitário de saúde quanto as suas atribuições e a falta de qualificação técnica desses profissionais sobre a temática podem comprometer a continuidade do cuidado e o controle da doença (Freitas, 2020; Sá et al., 2011).

Na perspectiva do SUS, Freitas e colaboradores (2017), as dificuldades operacionais do trabalho do ACS estão relacionadas à falta de formação adequada para o desenvolvimento de suas atribuições direcionadas às necessidades de saúde da população (como citado em, Cardozo-Gonzales et al., 2015).

Gaspar e colaboradores (2019), em estudo realizado em 2016 com os agentes comunitários de saúde afirma que a maioria (mais de 70\%) destes informou ser responsável por um número de famílias superior ao recomendado pelo MS, que é de 
até 150 famílias ou 750 pessoas, é reconhecidamente um fator que compromete a qualidade das ações prestadas por esse profissional, devido à quantidade de atividades e de cuidados específicos demandados por um número excessivo de moradores com diferentes agravos. Concluindo assim, que esse problema pode igualmente afetar a qualidade das ações desenvolvidas, o mesmo estudo mostrou que uma parcela considerável dos agentes comunitários de saúde possuía níveis insatisfatórios de conhecimento sobre tuberculose. Considerando-se a relação entre o conhecimento e as boas práticas sobre tuberculose.

Rizzo e colaboradores (2021, como citado em, Rocha et al. (2015) constatou que o nível de conhecimento dos agentes comunitários de saúde sobre tuberculose, medidas de controle e Tratamento Diretamente Observado (TOD) apresentaram lacunas com relação à forma de transmissão da doença, ao público-alvo do TOD e á técnica adequada para supervisão do tratamento.

Pesquisas apresentaram dificuldades gerais na atuação do agente comunitário de saúde em discernir quais são suas atribuições, mostrando ações que fogem à sua competência, a exemplo de agendamento de consultas e exames, entrega de encaminhamentos, medicamentos e recados da equipe para a comunidade e também trabalho administrativo e burocrático assim como o trabalho em área descoberta, a escassez de profissionais. Segundo Samúdio (2017), a não clareza das demandas da população, assim como a oferta de capacitações descontextualizadas e a falta de apoio das equipes da equipe de saúde da família, leva o agente comunitário de saúde a permitir a cobrança de ações incongruentes (como citado em, Ávila, 2011).

Batista (2018, como citado em, Maciel, 2008), afirma que deve ser alvo de reflexão, tratar da exposição de profissionais, com evidente carência de entendimento sobre a transmissão da tuberculose, a riscos de infecção sem a orientação devida e a provisão de equipamentos de proteção.

\section{Tema 3: Capacitação dos agentes comunitários de saúde}

Samúdio, (2017), refere que a educação permanente em saúde, como processo educativo em relação com o trabalho, oportuniza o aprendizado e não transmite informações, por isso pode operar mudanças no cuidado (como citado em, Ceccin, Bravin e Santos, 2011). A atuação dos agentes comunitários de saúde na atenção primaria a saúde exige qualificação adequada e permanente, para assumir suas atribuições junto à equipe de saúde da família e à comunidade.

O autor acima afirma ainda que investimentos são necessários no treinamento e manutenção da qualidade do trabalho, com supervisão e reflexão em equipe - assim, enfrentando os desafios com segurança e responsabilidade com o SUS. Talvez a escassez de discussões sobre o potencial do agente comunitário de saúde para exercer seu papel educador (Durão et al., 2011), além da formação, treinamento e capacitação oferecidos a esses profissionais, sejam os limitantes tanto no campo teórico e conceitual do

Segundo Morozini e colaboradores (2018), as formas de qualificação breves e intermitentes surgem como alternativa permanente demanda dos serviços por mais profissionais com condições de viabilizar suas inúmeras atividades. Assim, são tomadas como uma solução imediata, mas, no médio prazo, tornam-se um problema, pois nenhuma delas converge para uma profissionalização de fato, nem promove uma forma o consistente

Em estudo realizado por Freitas e colaboradores (2020), foi constatado que a maioria dos agentes comunitários consideram que o seu nível de conhecimento sobre a tuberculose precisa melhorar. É neste contexto que se inserem esses profissionais, alvos da intervenção educativa. Os achados apontam a relevância de garantir o conhecimento e habilidades específicas para o desempenho das funções desse profissional, pois a qualidade do cuidado fornecida pelo ACS é diretamente influenciada pela sua qualificação.

Contradizendo os outros estudos, Siqueira e colaboradores (2020), em estudo descritivo relata que os agentes comunitários de saúde consideraram serem mais (sempre) qualificados para atender tuberculose e trabalhar segundo a diversidade cultural da comunidade que os demais profissionais, ao passo que técnicos/auxiliares de enfermagem em menor peso (quase nunca) realizam educação permanente para o desenvolvimento de ações de controle da tuberculose. 
Acerca da formação, Siqueira e colaboradores (2020), afirma que os profissionais declararam que o tema tuberculose sempre foi abordado, com menor proporção (quase sempre) para enfermeiros e agentes comunitários ao analisar por categoria profissional. Apesar da diferenciação, a frequência mantém-se elevada e vai de encontro à literatura, que indica que os mesmos apresentam fragilidades no conhecimento sobre os sinais, sintomas e transmissão da doença, ainda que apresentem maior tempo de serviço e treinamento para atuar nas ações de controle e vigilância da Tuberculose.

Talvez a escassez de discussões sobre o potencial do agente comunitário de saúde para exercer seu papel educador (Freitas, 2020 como citado em, Durão et al, 2011), além da formação, treinamento e capacitação oferecidos a esses profissionais, sejam os limitantes tanto no campo teórico e conceitual do trabalho, como no detalhamento de suas atribuições (Freitas, et al., 2020).

\section{Considerações Finais}

Destarte, conclui-se que a contribuição do Agente Comunitário de Saúde no âmbito do acompanhamento da tuberculose na atenção primária a saúde, de acordo com os estudos levantados condescendem em diversos pontos sobre essa afirmativa. Os autores acima citados concordam com a importância e com as dificuldades apresentadas pelos estudos.

Foi possível identificar as dificuldades encontradas durante a atuação desses profissionais, o déficit na sua capacitação e no treinamento, o excesso de atividades, a incapacidade de conhecer suas atribuições e o desconhecimento sobre a tuberculose e as maneiras com as quais o agente comunitário enfrenta e se adaptam para atingir o portador de tuberculose. Com certeza, existe a necessidade de enfrentamento através de capacitação adequada, continua e permanente, treinamento e aquisição de conhecimento sobre a tuberculose desde o diagnóstico até o tratamento, deixando claro as atribuições e a forma como esse profissional deve supervisionar os pacientes de treinamento e aquisição de conhecimento a respeito da abordagem desses pacientes assim como a necessidade de obtenção de formas interativas e estratégicas de abordagem e acompanhamento ao portador de tuberculose.

Sendo assim se torna possível sugerir mudanças na relação paciente e agente comunitário de saúde, através de ações em saúde, com orientações temáticas, interdisciplinares e interpessoais, alinhadas com as diretrizes do SUS e com o olhar atento aos determinantes e condicionantes da saúde, com o intuito de promover ações e atingir o máximo de pacientes acometidos com a tuberculose, assim como seu acompanhamento durante o longo período de tratamento reduzindo assim o abandono e o número de óbitos.

Desta forma, cabe ressaltar a importância da educação em saúde, da capacitação, e da formação profissional dos agentes comunitários de saúde para melhorar a abordagem as questões acerca da tuberculose, promovendo o sucesso tanto na prevenção como para o tratamento.

Logo, deve-se considerar a realização de capacitações contínuas e permanentes aos Agentes Comunitários de Saúde, como intuito de propagar o conhecimento e capacitá-los para o enfrentamento a tuberculose e disseminação da informação sobre a patologia em tema, além de refletir sobre valorização deste profissional, que é fator de motivação para mudanças e melhorias na abordagem da comunidade e dos pacientes portadores de tuberculose.

\section{Referências}

Batista, L. D.A (2018) Capacitação de agentes comunitários de saúde de unidade básica de saúde como estratégia para detecção de casos de tuberculose. Fortaleza.

Brasil, (2019). Manual de Recomendações para o Controle da Tuberculose no Brasil. Ministério de Saúde: Brasília. https://bvsms.saude.gov.br/bvs/publicacoes/manual_recomendacoes_controle_tuberculose_brasil.pdf. 
Research, Society and Development, v. 11, n. 1, e4111124394, 2022

(CC BY 4.0) | ISSN 2525-3409 | DOI: http://dx.doi.org/10.33448/rsd-v11i1.24394

Barsil, (2020). Boletim epidemiológico da tuberculose 2020.https://www.saude.gov.br/images/pdf/2020/marco/24/Boletim-tuberculose-2020-marcas--1-.pdf.

Brasil. (2020). Data SUS tecnologia da informação. http://tabnet.datasus.gov.br/cgi/tabcgi.exe?sinannet/cnv/tubercma.def.

Fernandes., T. S, et al (2020). Estigma e preconceito na atualidade: vivência dos portadores de tuberculose em oficinas de terapia ocupacional. Physis: Revista de Saúde Coletiva, 30(1), e300103https://www.scielosp.org/article/physis/2020.v30n1/e300103/.

Freitas, P. R, Honda, E. Z, Pinto, E. S. G, Ferrier, M. R. L, \& Orfão, N. H. (2020) Intervenção educativa sobre tuberculose para agentes comunitários de saúde em unidades de atenção primária à saúde em um município da região Norte. Revista Desafios. Porto Velho- RO. 7(3). http://dx.doi.org/10.20873/uftv7-8556.

Gaspar, L. M. S. et al (2019). Conhecimento, atitudes e práticas de agentes comunitários de saúde sobre tuberculose pulmonar em uma capital do Nordeste do Brasil. Revista Ciência \& Saúde Coletiva, 24(10):3815-3824, 2019.

Marques, S, C. F (2011). As percepções dos pacientes em tratamento de tuberculose sobre sua doença: Uma Análise de Literária Científica. Rio de Janeiro.

Melo, L. S. O, et al (2020) Passos e descompassos no processo de cuidado aos portadores de tuberculose na atenção primária. Revista Enfermagem em Foco. 11 (1): $136-141$.

Morosine, M. V., \& Fonseca, A. M. Os agentes comunitários na Atenção Primária à Saúde no Brasil: inventário de conquistas e desafios. Revista Saúde Debate, $42,261-274$.

Rizzo, E. C et al (2021). Agentes sociais na Estratégia Saúde da Família (ESF) para o controle da Tuberculose: Educação permanente para os Agentes Comunitários de Saúde (ACS): uma revisão integrativa. https://downloads.editoracientifica.org/articles/210604974.pdf.

Siqueira T. C. et al (2020). O tratamento da tuberculose sob a ótica dos profissionais da Atenção Primária à Saúde. Revista Atenção Primária a Saúde.

Souza, M. T., Silva, D., \& Carvalho, R (2010). Revisão integrativa: o que é e como fazer. Revista Einstein. 8(1 Pt 1):1026.https://www.scielo.br/j/eins/a/ZQTBkVJZqcWrTT34cXLjtBx/?format=pdf\&lang=pt. 